\title{
Vitamin D and Pre-Eclampsia: Original Data, Systematic Review and Meta-Analysis
}

\author{
Elina Hyppönen ${ }^{\mathrm{a}-c}$ Alana Cavadino ${ }^{c}$ David Williams $^{\mathrm{d}}$ Abigail Fraser $^{\mathrm{e}}$ \\ Attila Vereczkey $^{g}$ William D. Fraser $^{f}$ Ferenc Bánhidy $^{\mathrm{h}}$ Deborah Lawlor ${ }^{\mathrm{e}}$ \\ Andrew E. Czeizeli
}

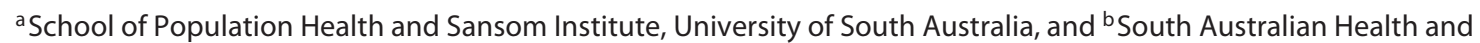
Medical Research Institute, Adelaide, S.A., Australia; ' ${ }^{C}$ Centre for Paediatric Epidemiology and Biostatistics, University College London Institute of Child Health, and 'Institute for Women's Health, University College Hospital, London, e MRC CAiTE Centre, School of Social and Community Medicine, University of Bristol, Bristol, and ${ }^{\mathrm{f}}$ Norwich Medical

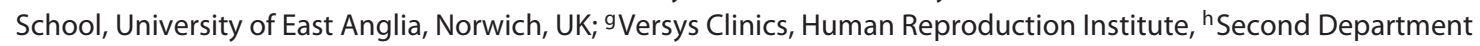
of Obstetrics and Gynecology, Semmelweis University, School of Medicine, and 'Foundation for the Community Control of Hereditary Diseases, Budapest, Hungary

\section{Key Words}

Vitamin D - 25-Hydroxyvitamin D · Supplementation .

Pre-eclampsia

\begin{abstract}
Background/Aims: Vitamin D may protect from pre-eclampsia through influences on immune modulation and vascular function. To evaluate the role of vitamin $D$ in the development of pre-eclampsia, we conducted a systematic review and meta-analysis including novel data from 2 largescale epidemiological studies. Methods: PubMed, EMBASE and the Cochrane Central Register of Controlled Trials were searched for prospective observational studies of association between vitamin $\mathrm{D}$ supplementation or status (measured by maternal 25 -hydroxyvitamin D, $25(\mathrm{OH}) \mathrm{D}$ ) with a subsequent risk of pre-eclampsia, or randomised controlled trials using vitamin D supplementation to prevent pre-eclampsia. The Hungarian Case-Control Surveillance of Con-
\end{abstract}

genital Abnormalities (HCCSCA) and the Avon Longitudinal Study of Parents and Children (ALSPAC) were included in meta-analyses with published studies. Results: Mothers receiving vitamin $D$ supplementation earlier in pregnancy had lower odds of pre-eclampsia [pooled odds ratios (OR) 0.81 and $95 \%$ confidence interval $(\mathrm{Cl}) 0.75-0.87, \mathrm{p}=2.4 \times$ $10^{-8}, 2$ studies] in the meta-analysis of published studies with HCCSCA. The meta-analysis of published studies with ALSPAC suggested an association between higher serum $25(\mathrm{OH}) \mathrm{D}$ levels and a reduced risk of pre-eclampsia (pooled OR 0.52 and $95 \% \mathrm{Cl} 0.30-0.89, \mathrm{p}=0.02,6$ studies). Randomised trials of supplementation were suggestive of protective association (pooled OR 0.66 and $95 \% \mathrm{Cl} 0.52-0.83$, $p=0.001,4$ studies). Conclusions: This study suggests that low maternal serum $25(\mathrm{OH}) \mathrm{D}$ concentrations increase preeclampsia risk and that vitamin $D$ supplementation lowers this risk. The quality of evidence is insufficient to determine a causal association, which highlights the need for adequately powered clinical trials.

\section{KARGER}

E-Mail karger@karger.com

www.karger.com/anm
(C) 2014 S. Karger AG, Basel

0250-6807/14/0634-0331\$39.50/0
Prof. Elina Hyppönen

School of Population Health

University of South Australia, GPO Box 2471

North Terrace, Adelaide, SA 5001 (Australia)

E-Mail elina.hypponen@unisa.edu.au 


\section{Introduction}

Pre-eclampsia is a life-threatening condition of pregnancy defined by the gestational onset of hypertension and proteinuria [1]. It affects $2-8 \%$ of first-time pregnant women, has marked international variation [2] and is responsible for up to $25 \%$ of maternal deaths worldwide [3]. Vitamin $\mathrm{D}$ is emerging as a promising agent for pre-eclampsia prevention [4]. Vitamin D deficiency is highly prevalent in women of reproductive age and in pregnant mothers [5]. If proven effective, the population level benefits of vitamin D supplementation would be substantial and likely to impact the long-term health of offspring [6].

Vitamin D is a seco-steroid pro-hormone which, for biological activation, undergoes two successive hydroxylations, firstly to 25-hydroxyvitamin D $(25(\mathrm{OH}) \mathrm{D})$, a nutritional biomarker for vitamin D status, and secondly to the active hormonal metabolite 1,25-dihydroxyvitamin D $\left(1,25(\mathrm{OH})_{2} \mathrm{D}\right)$, i.e. calcitriol. Calcitriol exerts the hormonal action via binding to nuclear vitamin $\mathrm{D}$ receptors, which are present throughout the body, including pregnancy-specific tissues such as the placenta and uterine placental bed (decidua). The placenta and decidua as well as other important target cells such as immune and endothelial cells have the molecular machinery for local production of calcitriol $[7,8]$.

Pre-eclampsia is thought to originate in early pregnancy when the maternal immune system limits placental invasion in mothers vulnerable to cardiovascular disease. Calcitriol can be considered a pregnancy-supporting factor [9] that could work through several mechanisms to reduce pre-eclampsia risk, including a direct influence of calcitriol on implantation, placental invasion and angiogenesis $[10,11]$. It is also believed to be important in directing immune responses by dendritic cells and macrophages at the fetal-placental interface as well as immunological adaptation by the mother to reduce the risk of infection and inflammation $[4,11]$.

Compared to normal pregnancies, vitamin D metabolism is markedly altered in pre-eclampsia. This may be due to reduced placental 1a-hydroxylase activity [12] resulting in lower circulating calcitriol concentrations compared to normotensive or chronically hypertensive pregnant women $[13,14]$. Vitamin D status is reportedly lower in pre-eclamptic mothers at the time of diagnosis [13, $15]$, but also before disease onset in some studies [16, 17].

In this paper, we investigate the association between vitamin $\mathrm{D}$ supplementation and status with pre-eclampsia risk in 2 large population studies: 59,789 participants from the Hungarian Case-Control Surveillance System of
Congenital Abnormalities (HCCSCA) and 5,058 participants from the Avon Longitudinal Study on Parents and Children (ALSPAC). We also undertook a systematic review and meta-analysis of all published prospective and intervention studies in order to evaluate the consistency and quality of evidence investigating an association between vitamin D supplementation and status in early pregnancy with pre-eclampsia risk.

\section{Methods}

Associations of vitamin D status during pregnancy with later pre-eclampsia were evaluated using novel data from 2 large-scale epidemiological studies. These data were incorporated into metaanalyses of previously published prospective and intervention studies that were identified following a systematic review.

\section{The Hungarian Case-Control Surveillance System of}

Congenital Abnormalities

The HCCSCA was carried out from 1980 to 1996 and consisted of 22,843 cases with congenital abnormality (CA) and 38,151 sex-, birth-week- and district-of-residence-matched controls with no CA $[18,19]$. Cases were identified through the Hungarian Congenital Abnormality Registry (coverage of approx. 90\%) and controls from the National Birth Registry of the Central Statistical Office. For the purpose of these analyses, we excluded mothers undergoing elective termination of pregnancy (102 case-mothers) or multiple pregnancies ( 421 case- and 410 control-mothers). Due to the suspected influences of antiepileptic mediation on vitamin D metabolism, those with diagnoses of epilepsy were also excluded (98 case- and 92 control-mothers) as well as 33 (14 case- and 19 control-mothers) due to missing data on the starting point for supplementation or pre-eclampsia onset and 53 (20 case- and 33 control-mothers) with pre-eclampsia onset recorded on or before the first 3 months of pregnancy. After these exclusions, there were 22,189 and 37,600 mothers of CA cases and controls, respectively, available for our analysis.

Information on maternal characteristics (including age, parity and employment), pregnancy complications, medications and use of supplemental vitamins was obtained from 'prenatal maternity logbooks' filled in at visits to prenatal care clinics $(21.0 \%$ of the case-mothers and $25.2 \%$ of the control-mothers), through retrospective structured questionnaires sent to the mother $(55.8 \%$ of case-mothers and $32.5 \%$ of control-mothers) or from concordant information from both sources (23.1\% of case-mothers and $42.4 \%$ of control-mothers) $[20,21]$. The majority of mothers returned their logbooks and questionnaires, with home visits done by regional nurses for all non-respondent case mothers. Nurses visited only 200 non-respondent and 600 respondent control mothers in 2 validation studies [22], as further follow-up was considered by the ethical committee to be disturbing to the parents of all healthy children. Nearly $100 \%$ of the pregnant women visited antenatalcare clinics, as both maternity allowance and maternity leave were conditional to these visits. The first visit was typically between the 6 th and 12th gestational week, with an average 7 visits per pregnancy. Overall, necessary information was available on $96.3 \%$ of cases $(84.4 \%$ from a reply to mailing and $11.9 \%$ from a nurse visit) 
and $83.0 \%$ of controls (82.6\% from a reply, and $0.4 \%$ from a visit). Information on maternal characteristics was available for all mothers with data on supplement intake. The Central Ethical Committee of the Hungarian Ministry of Health approved the methodology of the HCCSCA, the visits to non-respondent case mothers at home and the recording of personal data. Informed consent was signed by $98 \%$ of the mothers and the details on the remaining subjects were deleted, leading to their exclusion.

The routine vitamin $\mathrm{D}$ supplementation recommendation to all pregnant women was 3,000 IU/week (corresponding to $430 \mathrm{IU} /$ day) from the 20th gestational week. Several available multivitamin supplements also contained vitamin D (100-400 IU/day), and these were coded separately. All available single vitamin preparations contained cholecalciferol (vitamin $\mathrm{D}_{3}$ ), while multivitamins contained either cholecalciferol or ergocalciferol (vitamin $\mathrm{D}_{2}$ ). In our analyses, only mothers with a supplementation start time (recorded by gestational month) before the diagnosis of pre-eclampsia were considered. Maternal parity was based on the number of previous pregnancies, coded as $0,1-2$ or at least 3 . Maternal employment was coded as professional, managerial, skilled worker, semi-skilled worker, unskilled worker, housewife or other (student/retired/unemployed) [18].

Blood pressure (BP) and proteinuria (dipstick screening test) were measured during visits to prenatal-care clinics. After the 20th gestational week, all mothers with proteinuria were referred for a detailed laboratory examination. Proteinuria was defined as $>300$ $\mathrm{mg} / 24 \mathrm{~h}$, with pre-eclampsia diagnosed if accompanied by hypertension (at least two measures $>140 / 90 \mathrm{~mm} \mathrm{Hg}$ ).

\section{The Avon Longitudinal Study on Parents and Children}

The ALSPAC is a prospective population-based pregnancy cohort study that recruited 14,541 pregnancies resident in Avon, UK, with expected dates of delivery 1st April 1991 to 31st December 1992 (http://www.alspac.bris.ac.uk) [23]. There were 13,617 women with a live singleton birth who consented to have their obstetric data extracted from medical records. Ethical approval was obtained from the ALSPAC Law and Ethics Committee and the local Research Ethics Committee. For this study, we defined our eligible sample as women for whom we had sufficient information to determine the incidence of pre-eclampsia and for whom measures of $25(\mathrm{OH}) \mathrm{D}$ were available before diagnosis $(\mathrm{n}=5,058)$.

Information to determine incidence of pre-eclampsia was extracted from antenatal records by research midwives. The median number (interquartile range) of BP measurements in pregnancy was $14(11,16)$ and that of urine measurements was $11(10,14)$. Diagnosis of pre-eclampsia was based on the criteria of the International Society for the Study of Hypertension in Pregnancy [24] as: $\mathrm{BP} \geq 140 / \geq 90 \mathrm{~mm} \mathrm{Hg}$, measured on at least two occasions after 20 weeks of gestation, with proteinuria ( $\geq 300 \mathrm{mg} / 24 \mathrm{~h}$ ) occurring at the same time as the elevated BP. $25(\mathrm{OH}) \mathrm{D}_{3}$ and $25(\mathrm{OH}) \mathrm{D}_{2}$ concentrations in plasma or serum were measured with high-performance liquid chromatography tandem-mass spectrometry, with coefficients of variation for the assay $<10 \%$ across a working range of $1-250 \mathrm{ng} / \mathrm{ml}[25]$. Total $25(\mathrm{OH}) \mathrm{D}$ was calculated as the sum of $25(\mathrm{OH}) \mathrm{D}_{3}$ and $25(\mathrm{OH}) \mathrm{D}_{2}$.

Information on maternal age, ethnicity, education, physical activity and pre-pregnancy weight and height were obtained from questionnaire responses completed in early pregnancy. Ethnicity was categorized into white and non-white due to the small number of women from a non-white ethnic background. Education was categorised as: Certified Secondary Education [CSE, exams that used to be taken at age 16 by pupils considered less able than those taking ordinary O-level exams], vocational training, O-levels, Alevels or a university degree. Physical activity in pregnancy was assessed at 18 weeks gestation, expressed in average metabolic equivalents as previously described [26], and categorised into fifths. Pre-pregnancy BMI was calculated using a self-reported weight measurement. Self-reported maternal pre-pregnancy weight correlated highly with the first antenatal-clinic weight recorded in the medical records $(r=0.96)$.

\section{Search Strategy and Selection Criteria for Other Studies} Investigating Vitamin D Status and Supplementation in Relation to Pre-Eclampsia

An electronic search of academic, peer-reviewed journals was carried out using the PubMed central and EMBASE databases (up to 13 March 2013) to identify all studies on the association between vitamin $\mathrm{D}$ and pre-eclampsia published as full text papers in the English language. A search strategy was developed combining the results of the two main topics of interest, i.e. vitamin $\mathrm{D}$ and preeclampsia, and search terms were exploded using MeSH field tags (online suppl. fig. 2; see www.karger.com/doi/10.1159/000358338 for all online suppl. material). The Cochrane Central Register of Controlled Trials was also searched according to this strategy, and further records were identified by screening relevant texts for additional citations and searching the authors' personal archives and bibliographies.

Abstracts of all studies identified on-line by the search were evaluated independently by two review authors (A.C. and E.H.). Full texts of relevant abstracts were then assessed, with any discrepancies being resolved through discussion. Full texts were reviewed for eligibility if they met the following 3 main criteria: the study (1) observed pregnant women and recorded outcomes related to pre-eclampsia, (2) measured either serum 25(OH)D concentrations or recorded the use of vitamin $\mathrm{D}$ supplementation during pregnancy and (3) was either a clinical trial using oral vitamin D supplementation or a prospective observational study with information on $25(\mathrm{OH}) \mathrm{D}$ concentration or vitamin $\mathrm{D}$ supplementation obtained for a period preceding the diagnosis of pre-eclampsia. Studies measuring $1,25(\mathrm{OH})_{2} \mathrm{D}$ concentrations were not included since this active metabolite has a short half-life and is unsuitable for use as a biomarker for vitamin D intake/status [27].

\section{Data Extraction}

For relevant articles, the following information was recorded in a pre-defined spreadsheet: study design and population, geographical location of study, entry criteria, definition of pre-eclampsia, $25(\mathrm{OH}) \mathrm{D}$ assay used (if relevant) or frequency and dosage of vitamin D supplementation, month of $25(\mathrm{OH}) \mathrm{D}$ measurement/supplementation and any adjustments included in the final models. For intervention studies, information about further relevant factors was recorded, such as randomisation methods and blinding. Studies were classified into 3 groups, those investigating the prospective association between (1) vitamin D status (measured by $25(\mathrm{OH}) \mathrm{D})$ and incident pre-eclampsia, (2) vitamin D supplementation and incident pre-eclampsia and (3) interventions using vitamin D supplementation to prevent pre-eclampsia. 


\begin{tabular}{|c|c|}
\hline \multicolumn{2}{|c|}{$\begin{array}{l}\text { Titles and abstracts identified and screened for potential eligibility }(n=407) \\
\text { Records identified through PubMed search }(n=121) \\
\text { Records identified through EMBASE search }(n=280) \\
\text { Records identified in Cochrane Central Register of Controlled Trials }(n=3) \\
\text { Additional records identified through other sources }(n=3)\end{array}$} \\
\hline- & $\begin{array}{l}\text { Excluded based on title/abstract }(n=369) \\
\text { Review }(n=158) \\
\text { Non-relevant outcome or exposure }(n=63) \\
\text { Short communication/letter/conference abstract, etc. }(n=51) \\
\text { Case report }(n=12) \\
\text { Duplicate record }(n=85)\end{array}$ \\
\hline \multicolumn{2}{|c|}{ Full text assessed for eligibility $(n=38)$} \\
\hline$\longrightarrow$ & $\begin{array}{l}\text { Excluded based on full text }(n=25) \\
\text { Non-relevant outcome or exposure }(n=18) \\
\text { No comparison group }(n=2) \\
\text { Cross-sectional study }(n=4) \\
\text { Duplicated findings from another study }(n=1)\end{array}$ \\
\hline \multicolumn{2}{|c|}{$\begin{array}{l}\text { Included in review }(n=15) \\
\text { Intervention studies/clinical trials with supplements }(n=4) \\
\text { Prospective studies with supplements }((n=2) \\
\text { Prospective studies with } 25(O H) D(n=9)\end{array}$} \\
\hline \multicolumn{2}{|l|}{$\uparrow$} \\
\hline Novel data & from HCCSCA and ALSPAC studies $(n=2)$ \\
\hline
\end{tabular}

Fig. 1. Study selection process.

Statistical Analysis

In the HCCSCA, analyses were done separately for the CA case and control mothers. Vitamin D supplementation was considered only if taken before the diagnosis of pre-eclampsia, and was then analysed in dosage models comprising (1) 4 categories: no supplementation, multivitamin only, vitamin D supplementation only and vitamin $\mathrm{D}$ supplementation + multivitamin or higher dosage (>430 IU/day) in a single vitamin supplement and (2) 2 categories: no vitamin $\mathrm{D}$ supplementation versus any vitamin $\mathrm{D}$ supplementation. The main models used logistic regression adjusted for maternal age, parity and maternal employment. Information from other possible confounders such as BMI could not be included due to a lack of information. The likelihood ratio test or a trend test was used to calculate $\mathrm{p}$ values.

In the ALSPAC, vitamin D status was categorised as (1) $<37.5$ versus $\geq 37.5 \mathrm{nmol} / \mathrm{l},(2)<50$ versus $\geq 50 \mathrm{nmol} / \mathrm{l}$ and $(3) \geq 25$ to $<50$, $\geq 50-75$ and $\geq 75 \mathrm{nmol} / \mathrm{l}$. Models were run unadjusted and also adjusted for maternal age, BMI, ethnicity, physical activity, parity, education, gestational age at time of blood sampling for 25(OH)D and season of sampling (by trigonometric sine-cosine regression with the date of sampling). For women with more than one $25(\mathrm{OH}) \mathrm{D}$ pregnancy measure, the one taken earliest in pregnancy was chosen. Only measures before 20 weeks of pregnancy were included in the analyses.

To increase efficiency and minimise selection bias, multivariate multiple imputation was used to impute missing values of covariables for eligible women, including all exposures, covariables, outcomes and potential predictors of missing data (birth weight and gestational age at birth) in the imputation equations [28]. Switch- ing regression in STATA was used, as described by Royston [28]. Twenty cycles of regression switching were carried out and $30 \mathrm{im}$ putation datasets were generated. Distributions of imputed variables in the imputed datasets and the observed data (with no imputation) were very similar and the results of the complete case analyses were not substantively different from those of the multivariate multiple imputation, but were less precisely estimated.

\section{Data Synthesis}

Where appropriate, data from individual studies were pooled using fixed-effect meta-analyses with the Mantel and Haenszel method. Statistical heterogeneity was assessed using the $\mathrm{I}^{2}$ statistic. In the presence of heterogeneity, random-effects meta-analysis models were used with the method of DerSimonian and Laird.

When available from the original reports, odds ratios (OR) and $95 \%$ confidence intervals (CI) were used, and were otherwise calculated from other reported data. The direction of effect was reversed where appropriate, so that all OR related to the odds of pre-eclampsia in vitamin D-sufficient pregnant women compared to those who were considered insufficient (observational studies), or the odds of pre-eclampsia in the treatment group compared to the control group (intervention studies). To minimise bias, the main meta-analyses on observational studies of $25(\mathrm{OH}) \mathrm{D}$ and pre-eclampsia risk included only studies reporting confounder-adjusted OR. Meta-regression was used to explore possible variation in the association between $25(\mathrm{OH}) \mathrm{D}$ and pre-eclampsia by study size, trimester, assay type 


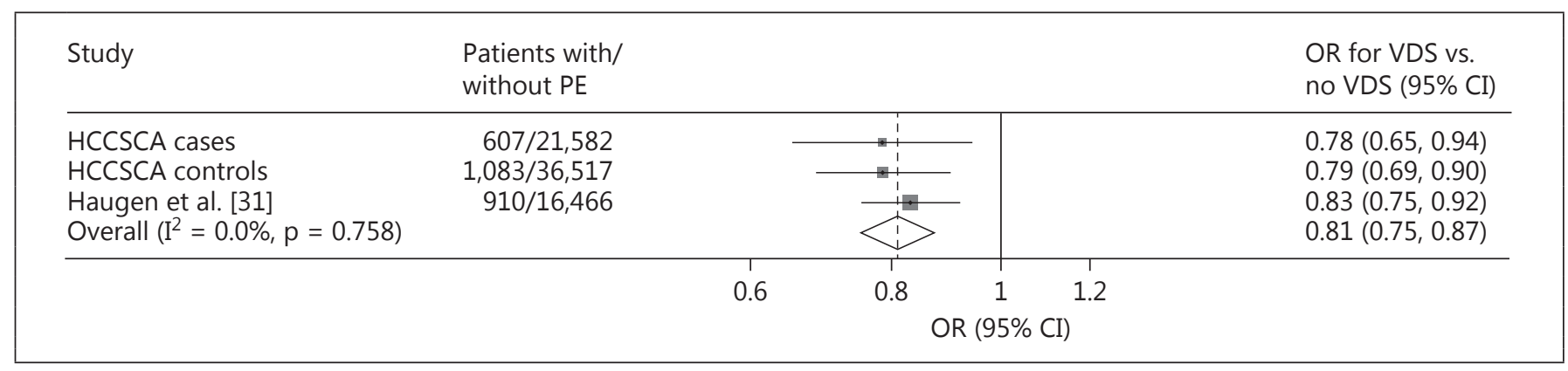

Fig. 2. Fixed effects meta-analysis on prospective observational studies of vitamin D supplementation (VDS) and subsequent risk of preeclampsia (PE).

Table 1. Vitamin D supplementation and risk of pre-eclampsia in the mothers from the HCCSCA $(n=59,789)$

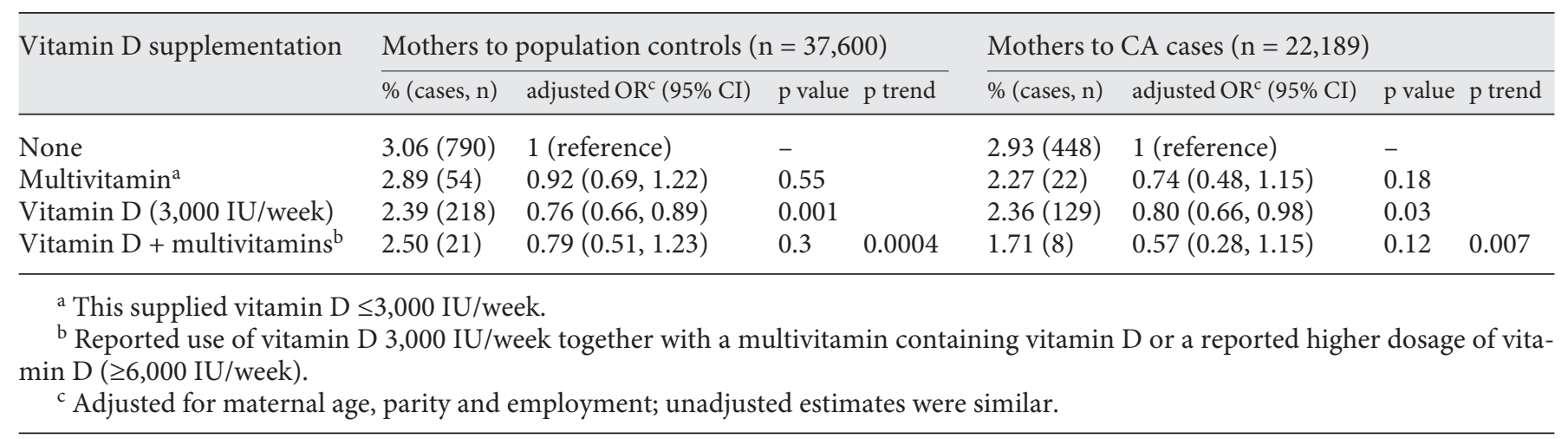

and latitude. Meta-analyses were also run separately for the two $25(\mathrm{OH}) \mathrm{D}$ thresholds $(<37.5$ and $50 \mathrm{nmol} / \mathrm{l})$, including the relevant ALSPAC results in both analyses. Further sensitivity analyses excluded studies on high-risk populations or studies of severe pre-eclampsia.

Publication bias was evaluated through the inspection of funnel plots and the statistical testing of funnel-plot asymmetry [29]. All analyses were carried out using STATA (version 12).

\section{Results}

The initial search of the PubMed central, EMBASE and Cochrane databases and references within published papers identified 407 potentially relevant unique abstracts and titles for review. Of these, 369 were excluded based on their abstract only, most of them being either reviews, not relevant or else records duplicated between databases (fig. 1). Of 38 papers that underwent a full text review, 18 were excluded due to a lack of relevant data on outcome or exposure, 2 had no comparison group and 1 had duplicated findings from another study. Four studies were excluded because only information on the cross-sectional differences in $25(\mathrm{OH}) \mathrm{D}$ concentrations was presented. The definition of pre-eclampsia was consistent across all studies, with most of them stating a requirement that systolic BP was $\geq 140 \mathrm{~mm} \mathrm{Hg}$ and/or diastolic BP was $\geq 90 \mathrm{~mm} \mathrm{Hg}$ and proteinuria was at least $300 \mathrm{mg} / 24 \mathrm{~h}$. In 2 of the studies $[17,30]$, the outcome considered was severe pre-eclampsia (online suppl. table 1).

\section{Observational Studies on Vitamin D Supplementation} and Risk of Pre-Eclampsia

In the HCCSCA, prevalence of pre-eclampsia was similar in mothers of CA cases and in controls (2.8 and 2.9\%, respectively). In both groups, vitamin D supplementation earlier in pregnancy was associated with a reduced risk of developing pre-eclampsia, with some evidence for a doseresponse effect (table 1).

We identified only 1 prospective observational study evaluating the association between vitamin D supple- 
Table 2. Maternal serum 25(OH)D concentrations before pre-eclampsia onset in the ALSPAC $(n=5,058)$

\begin{tabular}{lllll}
\hline & $\begin{array}{l}\text { Proportion in } \\
\text { category, } \%\end{array}$ & $\begin{array}{l}\text { Incidence of } \\
\text { pre-eclampsia, } \%\end{array}$ & $\begin{array}{l}\text { Unadjusted OR } \\
(95 \% \mathrm{CI})\end{array}$ & $\begin{array}{l}\text { Adjusted OR } \\
(95 \% \mathrm{CI})\end{array}$ \\
\hline$<25 \mathrm{nmol} / \mathrm{l}$ & 4.7 & 2.7 & $1.90(0.77,4.64)$ & $1.62(0.65,2.51)$ \\
$\geq 25$ to $<50 \mathrm{nmol} / 1$ & 30.2 & 2.3 & $1.60(0.88,2.88)$ & $1.44(0.79,2.63)$ \\
$\geq 50$ to $<75 \mathrm{nmol} / 1$ & 30.9 & 2.1 & $1.44(0.78,4.64)$ & $1.35(0.73,2.51)$ \\
$\geq 75 \mathrm{nmol} / 1$ & 34.2 & 1.4 & Ref & Ref \\
Per $10 \mathrm{nmol} / 1$ & & 1.9 & $0.93(0.86,1.01)$ & $0.95(0.88,1.02)$ \\
& & p trend $=0.06$ & p trend $=0.11$ \\
\end{tabular}

a Adjusted for maternal age, BMI, ethnicity, physical activity, parity, education, gestational age at the time of blood sampling for $25(\mathrm{OH}) \mathrm{D}$ and season of sampling.

mentation during pregnancy and pre-eclampsia risk [31]. The study compared mothers who had used vitamin D supplements before and/or during pregnancy with those who had not taken any vitamin D supplementation at any of these times, with an overall adjusted OR for preeclampsia of 0.83 (95\% CI $0.75-0.92)$ for supplementation in the 1st trimester of pregnancy, with or without previous or later supplementation. When HCCSCA was combined in a meta-analysis with the study of Haugen et al. [31], the overall estimate suggested $19 \%$ lower odds (95\% CI $25-13 \%, p=2.4 \times 10^{-8}$ ) of pre-eclampsia for mothers taking vitamin $\mathrm{D}$ supplements during pregnancy when averaged across dosages (fig. 2). The dosage of vitamin D in supplements taken by the mothers was $<600 \mathrm{IU} /$ day (most commonly, 200-430 IU/day) for the majority (>65\%) of women, in both the HCCSCA and the study by Haugen et al. [31].

\section{Observational Studies on 25(OH)D and Risk of Pre-Eclampsia}

In ALSPAC, there were 99 incident cases of preeclampsia. Pre-eclampsia incidence was the highest for mothers with $25(\mathrm{OH}) \mathrm{D}<25 \mathrm{nmol} / \mathrm{l}$ and lowest for those with $>75 \mathrm{nmol} / \mathrm{l}$; however, evidence of a linear trend was weakened after adjustment for confounders ( $p=0.11$; table 2).

From 8 independent reports for prospective association between $25(\mathrm{OH}) \mathrm{D}$ and pre-eclampsia, 3 provided unadjusted estimates only and were consequently excluded from the meta-analyses (online suppl. table 1) [32-35]. In the 5 published confounder-adjusted studies included in the analyses [16, 17, 30, 35-37], 3 different 25(OH)D assays were used, and the threshold for low vitamin $\mathrm{D}$ status was defined as $37.5 \mathrm{nmol} / \mathrm{l}$ in 2 studies and 50 $\mathrm{nmol} / \mathrm{l}$ in 3 studies. In 1 study, comparisons with the 'vi- tamin D sufficient' group (>75 nmol/l) were made. Two studies were of groups at a high risk of pre-eclampsia. All but 1 of the studies adjusted for season and BMI, with most of them also controlling for age, ethnicity, education or social class and gestational age for $25(\mathrm{OH}) \mathrm{D}$ assessment. 25(OH)D measures were done at the end of the 1st trimester in 1 study and other published studies included measures from the 2 nd trimester.

When the ALSPAC was added to a random effects meta-analysis of studies investigating a relationship between $25(\mathrm{OH}) \mathrm{D}$ and pre-eclampsia, mothers with higher concentrations of $25(\mathrm{OH}) \mathrm{D}$ (as defined in each study) showed a decreased odds of developing pre-eclampsia compared with those who had lower $25(\mathrm{OH}) \mathrm{D}$; pooled OR 0.52 (95\% CI 0.30-0.89, p = 0.02). Study-specific OR for the association between $25(\mathrm{OH}) \mathrm{D}$ and pre-eclampsia ranged from 0.19 to 0.82 (fig. 3). There was evidence of heterogeneity between the studies $\left(\mathrm{I}^{2}=60 \%, 95 \%\right.$ CI $4-84 \%$, pheterogeneity $=0.03$ ), with the association between $25(\mathrm{OH}) \mathrm{D}$ and pre-eclampsia seen in studies carried out at latitudes south from $45^{\circ} \mathrm{N}$ (all from the USA) but not in studies located $>45^{\circ} \mathrm{N}$ (OR 0.28 and 95\% CI 0.12 0.65 and OR 0.80 and $95 \%$ CI $0.56-1.13$, respectively, $\mathrm{p}_{\text {metaregression }}=0.04$; online suppl. fig. $3 \mathrm{~A}$ ). There were no systematic differences between studies supporting a stronger versus a weaker association in relation to study size, assay type or trimester ( $\mathrm{p}_{\text {metaregression }}>0.16$ for all comparisons; online suppl. fig. 3B-D). Estimated effect was similar both for studies using 37.5 and $50 \mathrm{nmol} / \mathrm{l}$ as the cut-off for low concentrations (pooled OR 0.66 and 95\% CI $0.32-1.35$ and OR 0.60 and 95\% CI 0.35-1.05, respectively) with precision reduced in both comparisons due to lower numbers (online suppl. fig. 4). Sensitivity analyses excluding one study of a high-risk population did not greatly affect the relationship between 


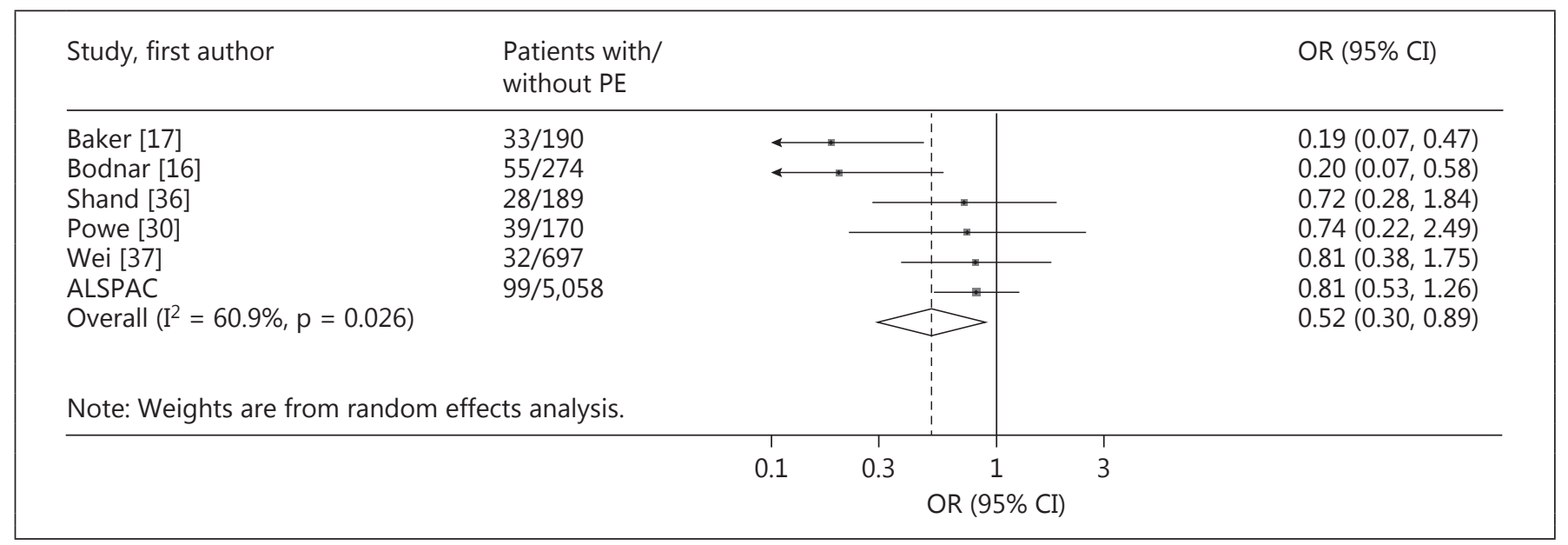

Fig. 3. Random effects meta-analysis on prospective observational studies of maternal serum $25(\mathrm{OH}) \mathrm{D}$ concentrations and subsequent risk of pre-eclampsia $(\mathrm{PE})$.

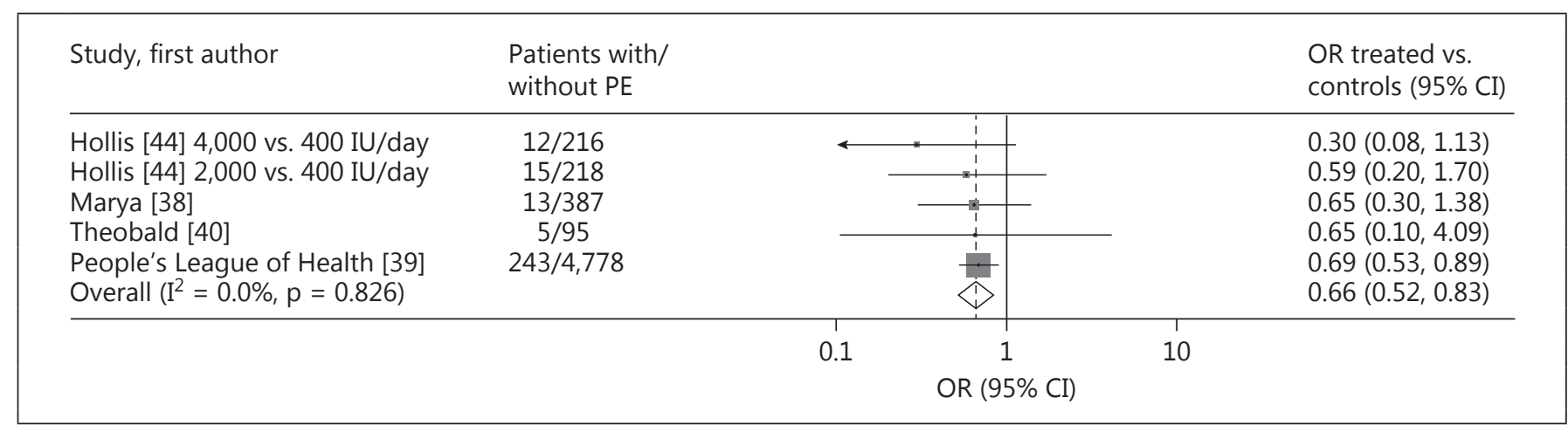

Fig. 4. Fixed effects meta-analysis on intervention studies using vitamin D supplementation to prevent pre-eclampsia (PE).

$25(\mathrm{OH}) \mathrm{D}$ and pre-eclampsia (pooled OR 0.48 and $95 \%$ CI $0.25-0.92, \mathrm{p}=0.03)$, while the observed association was attenuated (pooled OR 0.63 and 95\% CI 0.37-1.07, $\mathrm{p}=0.08$ ) when excluding 2 studies with the main outcome as severe pre-eclampsia. Effect estimates for all studies excluded from the meta-analyses due to lack of adjustment were suggestive of a protective association (OR 0.45-0.76). Examination of funnel plots did not provide any evidence for a publication bias $(\mathrm{p}=0.23$; online suppl. fig. 1).

\section{Randomised Trials on Vitamin D Supplementation and Pre-Eclampsia}

Of 4 trials included in the meta-analysis, 3 were placebo-controlled but unblinded studies [38-40]. The only blinded study [41] used supplementation with 400 IU/ day as the comparison group, with the treatment groups receiving 2,000 and 4,000 IU/day. In the other 3 studies, the dosage of vitamin $\mathrm{D}$ supplementation in the treatment group ranged from 450 to 1,000 IU/day, and all used a supplement including other micronutrients (all with calcium, 1 with vitamin A and 1 with a multivitamin).

Combining the data available from these 4 trials showed a reduced odds of pre-eclampsia in women taking vitamin $\mathrm{D}$ supplementation compared with the control group, with a pooled OR of 0.66 (95\% CI $0.52-0.83$, $\mathrm{p}=0.001$ ). Individual study OR ranged from 0.44 to 0.69 , with no statistical heterogeneity noted between the trials $\left(\mathrm{I}^{2}=0,95 \%\right.$ CI $0-48 \%$, pheterogeneity $=0.83$; fig. 4$)$. 


\section{Discussion}

The association between maternal vitamin D supplementation and status with the risk of pre-eclampsia was consistent across different types of studies, with evidence for benefits in prospective observational studies as well as in clinical trials. Two recent meta-analyses of observational studies also support an association between maternal serum $25(\mathrm{OH}) \mathrm{D}$ levels and pre-eclampsia, although in contrast to current study, neither of them restricted their analyses to confounder-adjusted studies and one of them included studies that measured concentrations at the time of pre-eclampsia $[42,43]$. Several studies provided evidence for a dose-dependent association, with the recent trial by Hollis and Wagner [44] providing the most notable example. However, the causality of the association remains unproven, as the bulk of evidence was obtained from observational studies and only 1 of the intervention studies was blinded. Pre-eclampsia is potentially a life-threatening condition for both mother and fetus. Delivery of the placenta remains the only cure, but is often associated with severe prematurity for the neonate. Vitamin D is a promising candidate for preeclampsia prevention, and there is an urgent need for well-controlled randomised trials to test its effectiveness and safety.

\section{Methodological Considerations}

In this meta-analysis, potential for publication bias was reduced by inclusion of unpublished as well as published data, with the decision to include 2 large unpublished studies made a priori to carrying out analyses. Assays and cut-off points or contrasts used to examine vitamin D 'deficiency' varied between studies included in the meta-analysis, making quantification of the magnitude of association difficult. In the HCCSCA, it can be argued that factors affecting pre-eclampsia risk differ between pregnancies leading to offspring with CA compared to those with healthy outcomes; however, the observed association between vitamin D supplementation and preeclampsia was similar in both sub-sets. Despite restricting our analysis to studies where measurements of vitamin D were obtained before the diagnosis of pre-eclampsia, we cannot discount the possible influence of reverse causation [i.e. the influence of underlying pre-eclampsia on $25(\mathrm{OH}) \mathrm{D}$ concentrations]. Measurement of vitamin D exposure was done in most studies during the 2 nd trimester, while only 1 study provided measures from the 1st trimester, and preconception measures were not available. Restriction of the sample to mothers with informa- tion on vitamin D supplementation reported before preeclampsia (compared to any stage of pregnancy) in the HCCSCA strengthened the association, which suggests that a false-positive association is unlikely. However, for studies assessing influences on pre-eclampsia risk by vitamin D status, where concentrations could be affected by less time spent outdoors due to the disease process, this would have been more likely to result in a type I error. With observational studies, it is not possible to exclude the possibility of residual confounding. While in the ALSPAC study we aimed for the inclusion of key confounding factors including physical activity (as a proxy for time spent outdoors), adjustments in previous publications were typically less comprehensive. Results from clinical trials remain inconclusive as most were of low quality, were not blinded and included other nutrients in addition to vitamin $\mathrm{D}$. While secondary analyses evaluating the association between vitamin $\mathrm{D}$ supplementation (as a single ingredient preparation) and pre-eclampsia risk in the recent double-blind, randomised trial were suggestive of a protective association [44], the numbers of cases were small and the outcome included mothers with gestational hypertension as well as pre-eclampsia.

\section{Mechanisms}

There are several mechanisms by which vitamin D could potentially prevent or at least delay the progression to pre-eclampsia. For example, progression to preeclampsia may result from a multitude of immune and vascular defects. One potential mechanism relates to a defective control of effector $\mathrm{T}$ cells by regulatory $\mathrm{T}$ cells. This can lead to poor placental invasion, which in turn leads to the release of placental-derived vasoconstrictor factors and consequent maternal hypertension and proteinuria [45]. Calcitriol is believed to be important in maintaining and restoring immune homeostasis and tolerance. Vitamin D receptors on immune cells express key enzymes involved in the hormonal activation (CYP27B1) and catabolism (CYP24A1) of vitamin D metabolites, suggesting that the availability and effectiveness of calcitriol can be directly regulated by the cells of the immune system [46]. The net result of calcitriol on adaptive immune responses leads to a skewing towards a more tolerogenic status, which is a maternal immune adaptation required for the maintenance of a healthy pregnancy [47]. In vitro studies have demonstrated that calcitriol administration leads to an up-regulation of regulatory $\mathrm{T}$ cell responses while proinflammatory responses are typically down-regulated [46], constituting an adaptation to maternal tolerance that would reduce the risk of pre-eclampsia. 
Vitamin D receptors on the heart and blood vessels suggest vitamin $\mathrm{D}$ has a cardio-protective effect, and calcitriol can influence endothelial and vascular smoothmuscle cell function as well as controlling inflammation and affecting the regulation of blood pressure through influences on the renin-angiotensin-aldosterone system [48]. Calcitriol may reduce pre-eclampsia risk through influences on angiogenesis [49]. This claim is supported by the known stimulatory effects of calcitriol on the expression of vascular endothelial growth factor in vascular smooth-muscle cells through vitamin $\mathrm{D}$ response elements in the vascular endothelial growth factor promoter [50]. Direct effects on the arterial wall by calcitriol may be important by preventing cholesterol uptake by macrophages and vascular smooth muscle proliferation; an athermanous pathology that is acutely observed in uteroplacental vessels in women with pre-eclampsia [51]. Therefore, vitamin $\mathrm{D}$ is likely to play an important role in the immune and cardiovascular changes necessary for a healthy pregnancy, highlighting the need to ensure that the existing public-health recommendations for vitamin $\mathrm{D}$ intake by pregnant mothers are endorsed.

\section{Conclusions}

This study has shown a consistent association between vitamin $\mathrm{D}$ and pre-eclampsia across different study types, supporting a role for vitamin $\mathrm{D}$ as a preventative agent against pre-eclampsia. However, due to the design and power of many of the trials included in our meta-analyses, this association is not conclusive and remains an impor- tant hypothesis that needs to be tested in a well-designed, randomised, controlled trial. If proven effective, vitamin $\mathrm{D}$ could provide cheap and safe prevention of preeclampsia. However, vitamin D deficiency is a correctable state that can affect the health of both mother and offspring also in other ways. While waiting for conclusive evidence that may support the proposed role for vitamin $\mathrm{D}$ in pre-eclampsia prevention, it is important to ensure that existing recommendations for vitamin D intake are systematically endorsed $[52,53]$.

\section{Acknowledgement}

We thank Erika Varga for the management of the HCCSCA's data. Funding for the project was provided by the British Heart Foundation (grant PG/09/023). Statistical analyses and work on ALSPAC were funded by the UK Medical Research Council (grants G0601653, G0701603 and SALVE/PREVMEDSYN with the Academy of Finland). D.W. receives part of his funding from the National Institute for Health Research University College London Hospitals Biomedical Research Centre. A.F. is funded by a UK Medical Research Council research fellowship (grant 0701594). The Centre for Paediatric Epidemiology and Biostatistics benefits from funding support from the MRC in its capacity as the MRC Centre of Epidemiology for Child Health. Research at the University College London Institute of Child Health and Great Ormond Street Hospital for Children NHS Trust is supported by R\&D funding received from the NHS Executive. The UK Medical Research Council (grant G074882), the Wellcome Trust (grant WT076467) and the University of Bristol provide core funding support for ALSPAC. The UK Medical Research Council (G0600705) and the University of Bristol provide core funding for the MRC Centre of Causal Analyses in Translational Epidemiology. The funders had no role in the study design, data collection and analysis, decision to publish or preparation of the manuscript.

\section{References}

$\checkmark 1$ Roberts JM, Cooper DW: Pathogenesis and genetics of pre-eclampsia. Lancet 2001;357: 53-56.

12 Duley L: The global impact of pre-eclampsia and eclampsia. Semin Perinatol 2009;33:130 137.

3 Khan KS, Wojdyla D, Say L, Gulmezoglu AM, Van Look PF: WHO analysis of causes of maternal death: a systematic review. Lancet 2006; 367:9.

4 Hyppönen E: Vitamin D for the prevention of preeclampsia? A hypothesis. Nutr Rev 2005; 63:225-232.

5 Holmes VA, Barnes MS, Alexander HD, McFaul P, Wallace JM: Vitamin D deficiency and insufficiency in pregnant women: a longitudinal study. Br J Nutr 2009;102:876-881.
6 6 Hyppönen E: Preventing vitamin D deficiency in pregnancy: importance for the mother and child. Ann Nutr Metab 2011;59:28-31.

$\checkmark 7$ Zehnder D, Evans KN, Kilby MD, Bulmer JN, Innes BA, Stewart PM, Hewison M: The ontogeny of 25-hydroxyvitamin $\mathrm{D}(3)$ 1alphahydroxylase expression in human placenta and decidua. Am J Pathol 2002;161:105-114.

-8 Barrera D, Avila E, Hernandez G, Mendez I, Gonzalez L, Halhali A, Larrea F, Morales A, Diaz L: Calcitriol affects hCG gene transcription in cultured human syncytiotrophoblasts. Reprod Biol Endocrinol 2008;6:3.

-9 Stumpf WE, Denny ME: Vitamin D (soltriol), light, and reproduction. Am J Obstet Gynecol 1989;161:1375-1384.
10 Zehnder D, Bland R, Chana RS, Wheeler DC, Howie AJ, Williams MC, Stewart PM, Hewison M: Synthesis of 1,25-dihydroxyvitamin $\mathrm{D}(3)$ by human endothelial cells is regulated by inflammatory cytokines: a novel autocrine determinant of vascular cell adhesion. J Am Soc Nephrol 2002;13:621-629.

11 Evans KN, Bulmer JN, Kilby MD, Hewison M: Vitamin D and placental-decidual function. J Soc Gynecol Investig 2004;11:263-271.

12 Diaz L, Arranz C, Avila E, Halhali A, Vilchis F, Larrea F: Expression and activity of 25-hydroxyvitamin D-1 alpha-hydroxylase are restricted in cultures of human syncytiotrophoblast cells from preeclamptic pregnancies. J Clin Endocrinol Metab 2002;87:3876-3882. 
13 Seely EW, Wood RJ, Brown EM, Graves SW: Lower serum ionized calcium and abnormal calciotropic hormone levels in preeclampsia. J Clin Endocrinol Metab 1992;74:1436-1440.

$\checkmark 14$ Halhali A, Tovar AR, Torres N, Bourges H, Garabedian M, Larrea F: Preeclampsia is associated with low circulating levels of insulinlike growth factor I and 1,25-dihydroxyvitamin D in maternal and umbilical cord compartments. J Clin Endocrinol Metab 2000;85: $1828-1833$.

-15 Robinson CJ, Alanis MC, Wagner CL, Hollis BW, Johnson DD: Plasma 25-hydroxyvitamin D levels in early-onset severe preeclampsia. Am J Obstet Gynecol 2010;203:366.e1-e6.

-16 Bodnar LM, Catov JM, Simhan HN, Holick MF, Powers RW, Roberts JM: Maternal vitamin $\mathrm{D}$ deficiency increases the risk of preeclampsia. J Clin Endocrinol Metab 2007;92: 3517-3522.

$\checkmark 17$ Baker AM, Haeri S, Camargo CA Jr, Espinola JA, Stuebe AM: A nested case-control study of midgestation vitamin $\mathrm{D}$ deficiency and risk of severe preeclampsia. J Clin Endocrinol Metab 2010;95:5105-5109.

$\checkmark 18$ Czeizel AE, Rockenbauer M, Siffel C, Varga E: Description and mission evaluation of the Hungarian case-control surveillance of congenital abnormalities, 1980-1996. Teratology 2001;63:176-185

19 Czeizel AE: First 25 years of the Hungarian congenital abnormality registry. Teratology 1997;55:299-305.

20 Czeizel AE, Petik D, Vargha P: Validation studies of drug exposures in pregnant women. Pharmacoepidemiol Drug Saf 2003;12:409_ 416.

21 Czeizel AE, Vargha P: Periconceptional folic acid/multivitamin supplementation and twin pregnancy. Am J Obstet Gynecol 2004;191: 790-794.

22 Cleve H, Bearn AG: Inherited variations in human serum proteins: studies on the groupspecific component. Ann NY Acad Sci 1961; 94:218-224.

-23 Fraser A, Macdonald-Wallis C, Tilling K, Boyd A, Golding J, Davey Smith G, Henderson J, Macleod J, Molloy L, Ness A, Ring S, Nelson SM, Lawlor DA: Cohort profile: the Avon Longitudinal Study of Parents and Children: ALSPAC mothers cohort. Int J Epidemiol 2012;42:97-110.

24 Davey DA, MacGillivray I: The classification and definition of the hypertensive disorders of pregnancy. Am J Obstetr Gynecol 1988; 158:892-898.

25 Tolppanen AM, Fraser A, Fraser WD, Lawlor DA: Risk factors for variation in 25-hydroxyvitamin $\mathrm{D}(3)$ and $\mathrm{D}(2)$ concentrations and vitamin $\mathrm{D}$ deficiency in children. J Clin Endocrinol Metabol 2012;97:1202-1210.
26 Fang Y, van Meurs JB, Arp P, van Leeuwen JP, Hofman A, Pols HA, Uitterlinden AG: Vitamin $\mathrm{D}$ binding protein genotype and osteoporosis. Calcif Tissue Int 2009;85:85-93.

27 Zerwekh JE: Blood biomarkers of vitamin D status. Am J Clin Nutr 2008;87:1087S-1091S.

28 Royston P: Multiple imputation of missing values. Stata J 2004;4:14

29 Egger M, Davey Smith G, Schneider M, Minder C: Bias in meta-analysis detected by a simple, graphical test. BMJ 1997;315:629-634.

30 Powe CE, Seely EW, Rana S, Bhan I, Ecker J, Karumanchi SA, Thadhani R: First trimester vitamin $\mathrm{D}$, vitamin $\mathrm{D}$ binding protein, and subsequent preeclampsia. Hypertension 2010;56:758-763.

-31 Haugen M, Brantsaeter AL, Trogstad L, Alexander J, Roth C, Magnus P, Meltzer HM: Vitamin $\mathrm{D}$ supplementation and reduced risk of preeclampsia in nulliparous women. Epidemiology 2009;20:720-726.

32 Azar M, Basu A, Jenkins AJ, Nankervis AJ, Hanssen KF, Scholz H, Henriksen T, Garg SK, Hammad SM, Scardo JA, Aston CE, Lyons TJ: Serum carotenoids and fat-soluble vitamins in women with type 1 diabetes and preeclampsia: a longitudinal study. Diabetes Care 2011;34:1258-1264.

- 33 Fernandez-Alonso AM, Dionis-Sanchez EC, Chedraui P, Gonzalez-Salmeron MD, PerezLopez FR: First-trimester maternal serum 25-hydroxyvitamin $\mathrm{D}(3)$ status and pregnancy outcome. Int J Gynaecol Obstet 2012;116: 6-9.

34 Yu CK, Ertl R, Skyfta E, Akolekar R, Nicolaides KH: Maternal serum vitamin D levels at 11-13 weeks of gestation in preeclampsia. J Hum Hypertens 2013;27:115-118.

35 Wetta L, Biggio J, Cliver S, Abramovici A, Barnes S, Tita A: Midtrimester vitamin D status is not associated with spontaneous preterm birth prior to 35 weeks. Am J Obstetr Gynecol 2012;206:S229-S230.

36 Shand AW, Nassar N, Von Dadelszen P, Innis SM, Green TJ: Maternal vitamin D status in pregnancy and adverse pregnancy outcomes in a group at high risk for pre-eclampsia. BJOG 2010;117:1593-1598.

37 Wei SQ AF, Hidiroglou N, Sarafin K, Julien P, Wu Y, Luo ZC, Fraser WD: Longitudinal vitamin $\mathrm{D}$ status in pregnancy and the risk of pre-eclampsia. Br J Obstetr Gynaecol 2012; 119:8.

38 Marya RK, Rathee S, Manrow M: Effect of calcium and vitamin D supplementation on toxaemia of pregnancy. Gynecol Obstet Invest 1987;24:38-42.

39 People's League of Health: The nutrition of expectant and nursing mothers in relation to maternal and infant mortality and morbidity. J Obstet Gynaecol Br Emp1946;53:498-509.
40 Theobald GW: Effect of calcium and vitamins $\mathrm{A}$ and $\mathrm{D}$ on incidence of pregnancy toxemia. Lancet 1937;1:1397-1399.

-41 Hollis BW, Johnson D, Hulsey TC, Ebeling M, Wagner CL: Vitamin D supplementation during pregnancy: double-blind, randomized clinical trial of safety and effectiveness. J Bone Miner Res 2011;26:2341-2357.

42 Aghajafari F, Nagulesapillai T, Ronksley PE, Tough SC, O'Beirne M, Rabi DM: Association between maternal serum 25-hydroxyvitamin $\mathrm{D}$ level and pregnancy and neonatal outcomes: systematic review and meta-analysis of observational studies. BMJ 2013;346:f1169.

43 Tabesh M, Salehi-Abargouei A, Esmaillzadeh A: Maternal vitamin D status and risk of preeclampsia: a systematic review and metaanalysis. J Clin Endocrinol Metabol 2013;98: 3165-3173.

-44 Hollis BW, Wagner CL: Vitamin D and pregnancy: skeletal effects, nonskeletal effects, and birth outcomes. Calcif Tissue Int 2013;92: 128-139.

45 Hsu P, Santner-Nanan B, Dahlstrom JE, Fadia M, Chandra A, Peek M, Nanan R: Altered decidual DC-SIGN+ antigen-presenting cells and impaired regulatory $\mathrm{T}$-cell induction in preeclampsia. Am J Pathol 2012;181:21492160.

46 Hewison M: Vitamin D and immune function: autocrine, paracrine or endocrine? Scand J Clin Lab Invest 2012;243(suppl ):92102.

47 Erlebacher A: Mechanisms of T cell tolerance towards the allogeneic fetus. Nat Rev Immunol 2013;13:23-33.

48 Li YC, Kong J, Wei M, Chen ZF, Liu SQ, Cao LP: 1,25 -Dihydroxyvitamin $\mathrm{D}(3)$ is a negative endocrine regulator of the renin-angiotensin system. J Clin Invest 2002;110:229-238.

49 Grundmann M, Haidar M, Placzko S, Niendorf R, Darashchonak N, Hubel CA, von Versen-Hoynck F: Vitamin D improves the angiogenic properties of endothelial progenitor cells. Am J Physiol Cell Physiol 2012; 303:C954-C962.

50 Cardus A, Panizo S, Encinas M, Dolcet X, Gallego C, Aldea M, Fernandez E, Valdivielso JM: 1,25-dihydroxyvitamin D3 regulates VEGF production through a vitamin $D$ response element in the VEGF promoter. Atherosclerosis 2009;204:85-89.

51 Brosens JJ PR, Brosens IA: The myometrial junctional zone spiral arteries in normal and abnormal pregnancies: a review of the literature. Am J Obstetr Gynecol 2002;187:8.

52 Ross et al (eds): Dietary Reference Intakes for Calcium and Vitamin D. Washington, National Academy of Sciences, 2011.

53 Hyppönen E, Boucher BJ: Avoidance of vitamin D deficiency in pregnancy in the United Kingdom: the case for a unified approach in national policy. Br J Nutr 2010;104:309-314. 